\title{
Reduction of Radiation Exposure by Modifying Imaging Manner and Fluoroscopic Settings during Percutaneous Pedicle Screw Insertion
}

\author{
Hyun Jun Kim, Eun Soo Park, ${ }^{1}$ Sang Ho Lee, ${ }^{2}$ Chan Hong Park, ${ }^{3}$ Seok Won Chung ${ }^{1}$ \\ Department of Neurosurgery, ${ }^{\dagger}$ Daegu Wooridul Spine Hospital, Daegu, Korea \\ Department of Neurosurgery, ${ }^{2}$ Wooridul Spine Hospiral, Seoul, Korea \\ Department of Anesthesiology and Pain Medicine, ${ }^{3}$ Daegu Wooridul Spine Hospital, Daegu, Korea
}

Objective : Percutaneous pedicle screw (PPS) fixation is a needle based procedure that requires fluoroscopic image guidance. Consequently, radiation exposure is inevitable for patients, surgeons, and operation room staff. We hypothesize that reducing the production of radiation emission will result in reduced radiation exposure for everyone in the operation room. Research was performed to evaluate reduction of radiation exposure by modifying imaging manner and mode of radiation source.

Methods : A total of 170 patients (680 screws) who underwent fusion surgery with PPS fixation from September 2019 to March 2020 were analyzed in this study. Personal dosimeters (Polimaster Ltd.) were worn at the collar outside a lead apron to measure radiation exposure. Patients were assigned to four groups based on imaging manner of fluoroscopy and radiation modification (pulse mode with reduced dose) : continuous use without radiation modification (group 1, n=34), intermittent use without radiation modification (group 2, $\mathrm{n}=54$ ), continuous use with radiation modification (group $3, \mathrm{n}=26$ ), and intermittent use with radiation modification (group 4, $\mathrm{n}=56$ ). Post hoc Tukey Honest significant difference test was used for individual comparisons of radiation exposure/screw and fluoroscopic time/screw.

Results : The average radiation exposure/screw was $71.45 \pm 45.75 \mu \mathrm{Sv} / \mathrm{screw}$ for group 1, 18.77 $\pm 11.51 \mu \mathrm{Sv} / \mathrm{screw}$ for group 2 , $19.58 \pm 7.00 \mu \mathrm{Sv} / \mathrm{screw}$ for group 3, and $4.26 \pm 2.89 \mu \mathrm{Sv} / \mathrm{screw}$ for group 4. By changing imaging manner from continuous multiple shot to intermittent single shot, $73.7 \%$ radiation reduction was achieved in the no radiation modification groups (groups 1,2 ), and $78.2 \%$ radiation reduction was achieved in the radiation modification groups (groups 3,4). Radiation source modification from continuous mode with standard dose to pulse mode with reduced dose resulted in $72.6 \%$ radiation reduction in continuous imaging groups (groups 1,3) and $77.3 \%$ radiation reduction in intermittent imaging groups (groups 2, 4). The average radiation exposure/screw was reduced $94.1 \%$ by changing imaging manner and modifying radiation source from continuous imaging with standard fluoroscopy setting (group 1) to intermittent imaging with modified fluoroscopy setting (group 4). A total of 680 screws were reviewed postoperatively, and $99.3 \%$ (675) were evaluated as pedicle breach grade $0(<2 \mathrm{~mm})$.

Conclusion : The average radiation exposure/screw for a spinal surgeon can be reduced $94.1 \%$ by changing imaging manner and modifying radiation source from real-time imaging with standard dose to intermittent imaging with modified dose. These modifications can be instantly applied to any procedure using fluoroscopic guidance and may reduce the overall radiation exposure of spine surgeons.

Key Words : Radiation dosage · Minimally invasive surgical procedures · Pedicle screw · Radiation exposure · Fluoroscopy.

- Received : December 8, 2020 •Revised : February 9, 2021 •Accepted : March 26, 2021

- Address for reprints : Eun Soo Park

Department of Neurosurgery, Daegu Wooridul Spine Hospital, 648 Gukchaebosang-ro, Jung-gu, Daegu 41939, Korea

Tel : +82-53-212-3000, Fax : +82-53-212-3049, E-mail : seamannn@naver.com, ORCID : https://orcid.org/0000-0002-1628-5296

This is an Open Access article distributed under the terms of the Creative Commons Attribution Non-Commercial License (http://creativecommons.org/licenses/by-nc/4.0) which permits unrestricted non-commercial use, distribution, and reproduction in any medium, provided the original work is properly cited. 


\section{INTRODUCTION}

Short segment fixation with transpedicular screw is a widely used technique to stabilize degenerative spondylolisthesis or traumatic spinal fractures. Percutaneous pedicle screw (PPS) fixation is a widely used technique to overcome the disadvantages of open pedicle screw fixation, such as higher infection rate, greater blood loss, more damage to paraspinal musculature, prolonged operation time, and longer hospital stay ${ }^{20)}$. PPS is also valuable as the anterior lateral approach of interbody cage insertion technique has become popular. However, PPS is a needle based procedure that requires fluoroscopic image guidance. Consequently, radiation exposure is inevitable for the patient, surgeon, and operation room staff.

Gamma rays and X-rays are the most frequently used ionizing radiation in the medical field and have been classified as carcinogens by the World Health Organization ${ }^{7}$. The National Council on Radiation Protection and Measurements and the International Commission on Radiological Protection (ICRP) has published the maximal limits of occupational exposure as $20 \mathrm{mSv}$ per year averaged over 5 years, or a maximum of 50 $\mathrm{mSv} /$ year in any 1 year for radiation workers, including spine surgeons $^{19)}$. The limit is $1 \mathrm{mSv} /$ year for non-radiation workers, and this can also apply to patients. These suggested limits are not recommended doses, since there is no safe level of radiation exposure. The general philosophy of the ICRP guidelines is to maintain radiation exposure "as low as reasonably achievable”. Therefore, every effort should be made to reduce the radiation exposure of radiation workers and patients.

Even routine image studies prior to fusion surgery, such as computed tomography (CT) scan of 5-6 vertebral levels and simple chest X-ray, involve $6-7 \mathrm{mSv}$ and $0.1 \mathrm{mSv}$ of radiation exposure, respectively ${ }^{14,16)}$. These exposures already exceed the annual recommend exposure level for non-radiation workers. Therefore, to reduce total exposure during care, intraoperative radiation exposure should be reduced not only for operative staff but also for patients. To achieve this goal, modification of fluoroscopic mode and current has been attempted in many other medical fields, including cardiovascular interventional electrophysiology and lumbar transforaminal epidural steroid injections ${ }^{3,9)}$. Godzik et al. ${ }^{6}$ also applied this modification in their prospective cohort study of minimally invasive spine surgery. These studies reported significant radiation reduction by setting fluoroscopy to pulse mode and reducing radiation output ( $\mathrm{kV}$ and $\mathrm{mA}$ values). Total fluoroscopic time also influences total radiation exposure. Intermittent single-shot imaging, rather than real-time multiple-shot imaging, is generally recommended to minimize radiation exposure imaging ${ }^{18)}$. In this study, we evaluate the effect of reducing radiation exposure by modifying imaging manner and mode of radiation source.

\section{MATERIALS AND METHODS}

\section{Study design, patient population, procedure indi- cation, and inclusion criteria}

This retrospective clinical study was performed in a single hospital. The study was approved by the Institutional Review Board of Wooridul Spine Hospital (IRB\# 2020-04-WSH-008), and all patients gave informed consent before enrollment. The study was conducted in 246 patients who underwent fusion surgery with PPS fixation from May 2019 to March 2020. PPS was performed with either the Sextant system (Medtronic Inc., Memphis, TN, USA) or the Viper system (Johnson \& Johnson Medical, Edison, NJ, USA). Procedures were performed using fluoroscopic image guidance in either continuous mode with standard dose or pulse mode with reduced dose.

Fusion surgery was indicated in patients with 1) higher than grade 1 spondylolisthesis with dynamic instability, 2) concomitant central and extra-foraminal intervertebral disc herniation who required total facetectomy, or 3) bilateral foraminal stenosis with isthmic defect. To unify the surgical techniques, only patients who underwent a single level lumbar interbody fusion fixed by sextant screw system were included in this study.

A spine surgeon in fellowship training performed all fixation procedures using the same C-arm fluoroscopy unit (Simens Arcadis Orbic; Simens AG, Muenchen, Germany). To minimize experience-based bias, the first 40 cases (176 screws) were excluded in this study. Nineteen additional multi-level fixation cases (118 screws) and 17 cases (68 screws) performed by the Viper system were excluded. Consequently, 170 cases (680 screws) were included in the final analysis. Patients were assigned to four groups based on fluoroscopy imaging manner and radiation modification (pulse mode with reduced dose) : real-time use without radiation modification (group 1), intermittent use without radiation modification (group 2), re- 
al-time use with radiation modification (group 3), and intermittent use with radiation modification (group 4) (Fig. 1) The term 'real-time' defined as continuous display of fluoroscopy, and 'intermittent' defined as interrupted display of fluoroscopy.

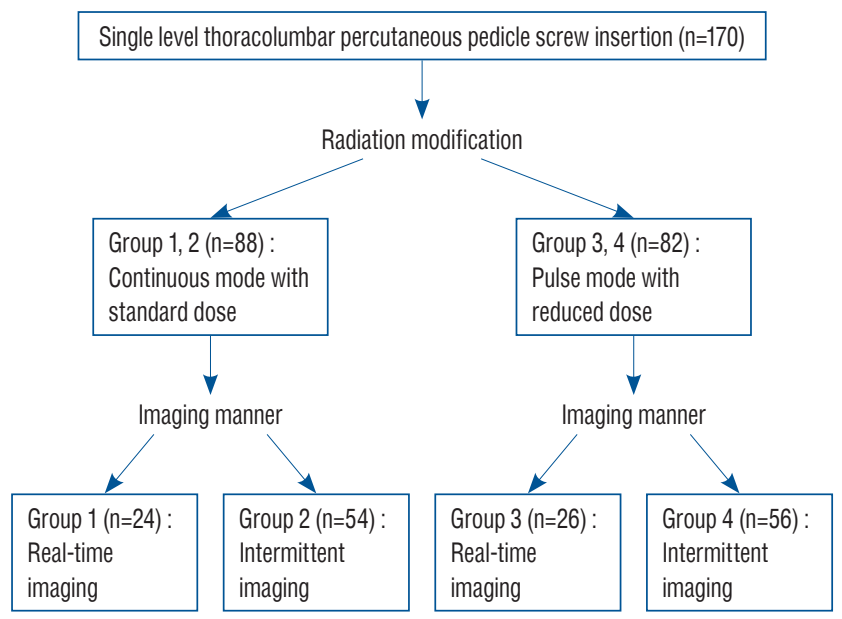

Fig. 1. Flow-chart of classification criteria for groups 1, 2, 3, and 4. Patients were grouped based on radiation modification and imaging manner.

\section{Demographics}

Demographic data was collected from patient medical charts. Sex, age, height, weight, body mass index (BMI), past medical history, operation level, and fusion type were recorded as demographic data. Anterior lumbar interbody fusion was classified as the anterior group, pre-psoas oblique and trans-psoas direct lateral lumbar interbody fusion were classified as the lateral group, and trans-foraminal and posterior lumbar interbody fusion were classified as the posterior group. Underlying radio-sensitive diseases were reviewed, including malignancy, thyroid disease (hyper- or hypothyroidism), and cataract.

\section{Surgical technique, radiation modification, and measurement}

After anterior or lateral approach interbody cage insertion, patients were placed in the prone position on a radiolucent Wilson's table under general anesthesia for PPS insertion. The procedure was performed in the same position for patients who underwent posterior approach interbody cage insertion.
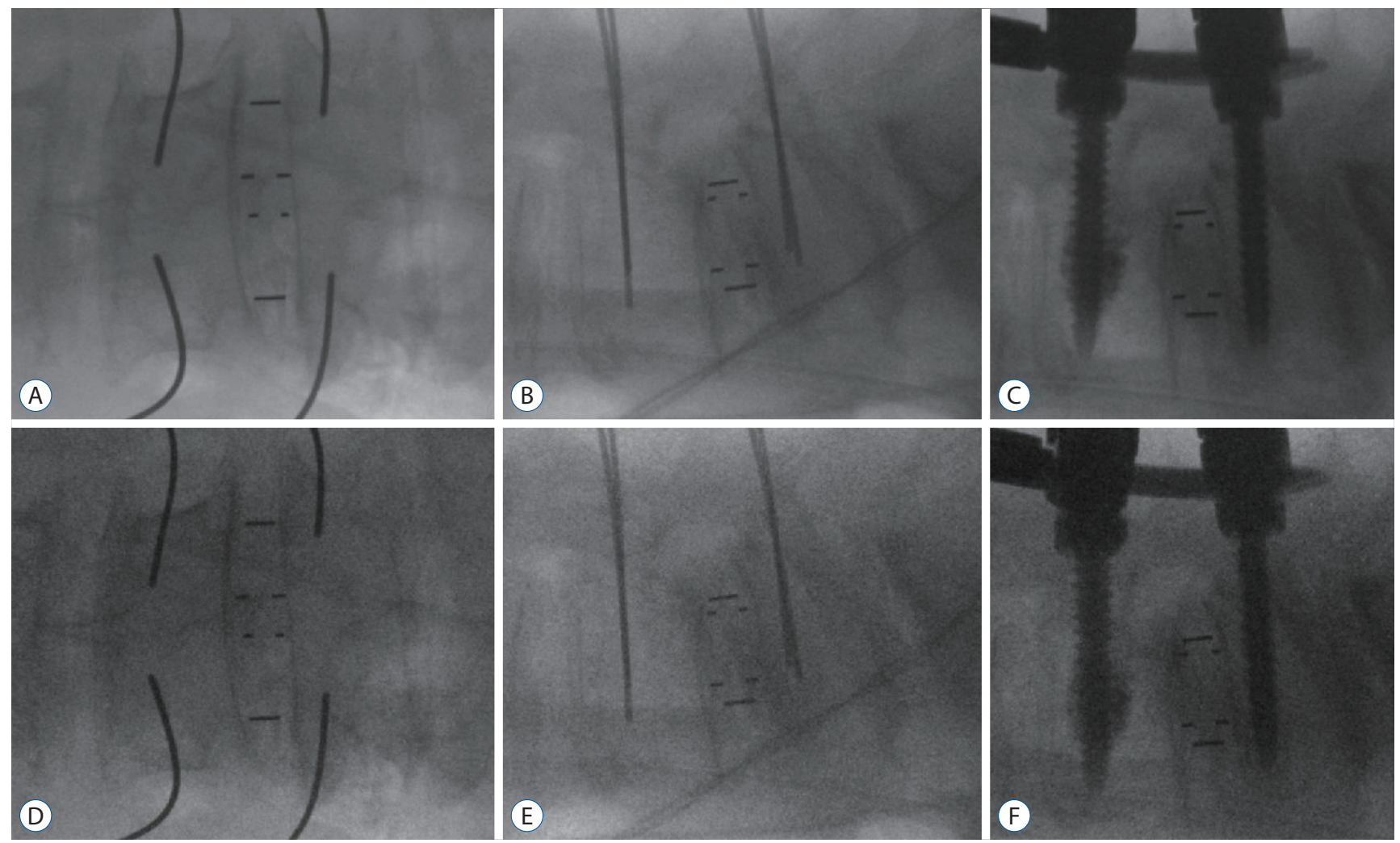

Fig. 2. Anteroposterior (A) and lateral (B) images were obtained after K-wire placement followed by rod insertion (C) using continuous mode with standard dose fluoroscopy. After modifying radiation source to pulse mode with reduced dose, image quality decreased slightly (D-F). 
During the procedure, a single plane C-arm fluoroscopy unit was used for intraoperative imaging. After confirmation of the surgical level in anteroposterior (AP) and lateral fluoroscopic images, the midline, lateral border of pedicles, and Jamshidi needle insertion points were marked on the skin.

Radiation exposure measurement began with confirmation of the skin incision point by Jamshidi needle. Personal dosimeters (PM1621 personal dosimeter; Polimaster Ltd., Minsk, Republic of Belarus) were worn at the collar outside of lead aprons. After a $1.5-2.0 \mathrm{~cm}$ skin incision was made, the Jamshidi needle was placed on the outer margin of the superior articular process and transverse process junction. Proper needle placement was evaluated by AP fluoroscopic image. Then, the needle was advanced until it was close to the medial margin of the pedicle. The $\mathrm{C}$-arm was then moved to the lateral angle and the needle was further advanced for more than half of the vertebral body depth. A linear guide wire was then placed through the inside hole of the Jamshidi needle. After wire placement, tapping and placement of the real screw, rod connection, and final capping were performed with lateral fluoroscopic inspection.

Procedures of groups 1 and 2 were performed under continuous mode with standard dose using an automatic setting of the $\mathrm{C}$-arm machine. The continuous mode had a frequency of 30 fractions/second, and dose was 70 to $90 \mathrm{kV}$ with 4 to $6 \mathrm{~mA}$ at the AP position and 95 to $110 \mathrm{kV}$ with 5 to $6 \mathrm{~mA}$ at the lateral position. For groups 3 and 4 , frequency was changed to pulse mode ( 8 fractions/second) and dose was reduced by $2-5$
$\mathrm{kV}$ while maintaining original $\mathrm{mA}$ value. Dose was reduced until the cortical bone margin of the pedicle and the vertebral body were visible with proper clearance to perform the procedure. Fluoroscopic images of standard and reduced radiation dose were demonstrated on Fig. 2. Although it showed blurred quality of images in reduced dose pictures (Fig. 2D-F), major anatomic structures such as pedicle and facet joints are still well visible compared to images of standard dose (Fig. 2A-C). Fluoroscopy was routinely used in an intermittent single shot manner (groups 2 and 4). However, if the location of the needle, wire, or taper was uncertain, fluoroscopy was changed to realtime multiple shot mode to avoid any pedicle invasion (groups 1 and 3). Therefore Measurement of radiation exposure finished after final confirmation of proper PPS location and rod connection state.

\section{Preoperative radiologic evaluation, intraopera- tive information, and radiation exposure}

Bone mineral density (BMD) was assessed in every patient before fusion surgery to evaluate bone quality. Preoperative radiologic evaluation was performed on preoperative $\mathrm{CT}$, and the existence of sclerosis, small pedicle, and facet joint hypertrophy was evaluated. These radiologic features are considered obstacles for PPS insertion because they obstruct passage of the bone needle and cover the needle insertion point. Patients with these features require more frequent fluoroscopic imaging to confirm correct instrument insertion. Sclerosis was defined as cancellous bone with similar density to cortical bone
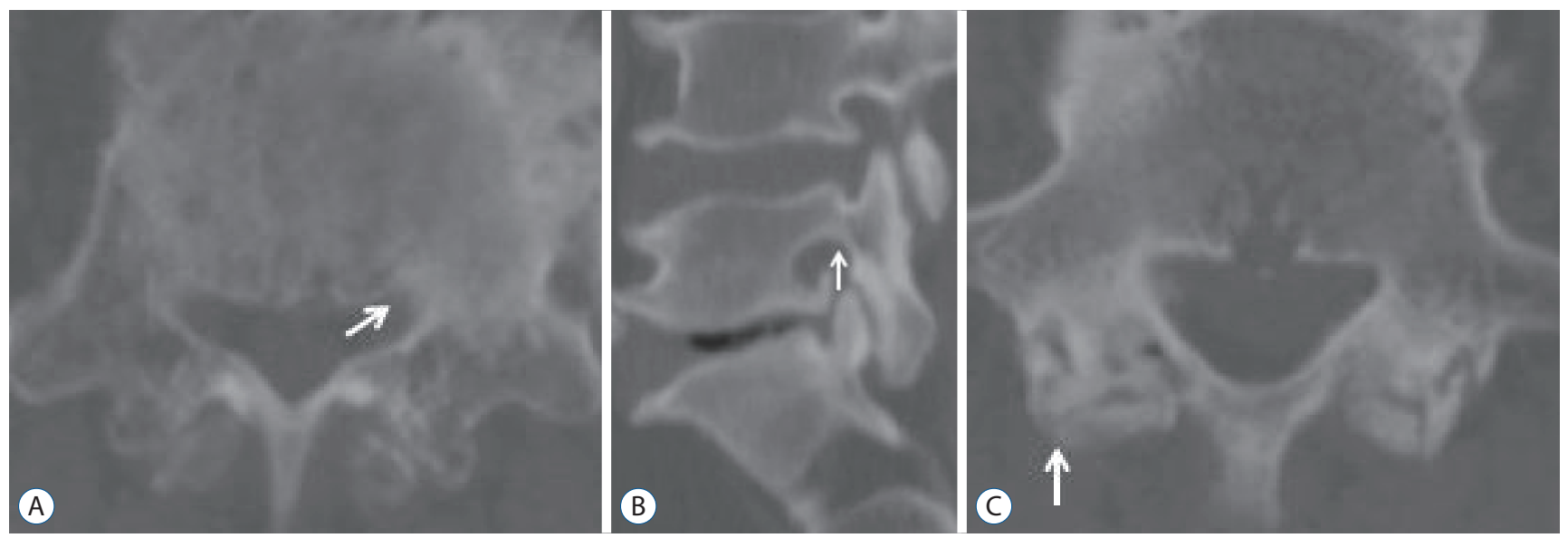

Fig. 3. Three anatomic features interfere with percutaneous pedicle screw insertion. Rigid sclerosis with high bone density on preoperative computed tomography is visible on left L4 pedicle and vertebral body (white arrow) (A). Pedicle with vertical diameter of $5.3 \mathrm{~mm}$ is visible on left L5 peidcle (white arrow) (B). Bilateral facet joint hypertrophy is visible on both L4-5 facet joints (white arrow) (C). 
(Fig. 3A). Small pedicle was defined as a pedicle with vertical or horizontal diameter less than $5.5 \mathrm{~mm}$ (Fig. 3B). Facet joint hypertrophy was defined as the existence of degenerative bony overgrowth invading the PPS insertion point (Fig. 3C).

Operation time was assessed from the beginning of Jamshidi needle insertion to removal of all sextant screw systems. Cement augmentation was performed for osteoporotic bone or whenever screw loosening was detected after reduction procedure. Operation time and number of cases of cement augmentation were counted as intraoperative information. Radiation was measured by personal dosimeter in units of $\mu \mathrm{Sv}$. Fluoroscopic time was recorded by the C-arm machine. These values were divided by the number of screws. Therefore the radiation exposure per screw $(\mu \mathrm{Sv} / \mathrm{screw})$ can be calculated from the result of this study. As $20 \mathrm{mSv}$ is recommended radiation exposure limit per year for radiation workers by ICRP, the number of screws to reach the annual limit of $20 \mathrm{mSv}$ can be calculated by dividing $20 \mathrm{mSv}$ by $\mu \mathrm{Sv} / \mathrm{screw}$.

\section{Postoperative radiologic outcome}

Postoperative radiologic evaluation was performed on postoperative magnetic resonance image (MRI), and the distance and direction of pedicle breach were evaluated. Breach was divided to 3 grade categories defined as follows : grade 0 (within $2.0 \mathrm{~mm}$ ), grade $1(2.0-4.0 \mathrm{~mm})$, and grade $2(4.0-6.0 \mathrm{~mm})^{5}$. Three evaluators graded breach grade, and the highest grade was selected as the final breach grade to avoid underestimation. The length of breach was measured by each operator, the first author, and the corresponding author of this article. The measurements of the three investigators had an intra-class correlation of $96.2 \%$ with statistical significance $(p<0.001)$. The mean value of the three measurements was obtained to further adjust the errors between the investigators. Complications related to pedicle breach during the postoperative care period were also investigated.

\section{Statistics}

Demographics, preoperative radiologic evaluation, intraoperative information, radiation exposure, and radiologic out-

Table 1. Demographics

\begin{tabular}{|c|c|c|c|c|c|}
\hline & Group $1(n=34)$ & Group $2(n=54)$ & Group $3(n=26)$ & Group $4(n=56)$ & $p$-value \\
\hline Sex & & & & & 0.784 \\
\hline Female & 20 & 35 & 16 & 31 & \\
\hline Male & 14 & 19 & 10 & 25 & \\
\hline Age (years) & $66.00 \pm 10.62$ & $64.06 \pm 10.55$ & $63.46 \pm 7.49$ & $65.43 \pm 9.29$ & 0.671 \\
\hline Height (cm) & $158.53 \pm 9.54$ & $158.76 \pm 9.35$ & $159.38 \pm 8.21$ & $157.32 \pm 8.79$ & 0.756 \\
\hline Weight (kg) & $62.74 \pm 11.84$ & $62.57 \pm 12.25$ & $63.15 \pm 9.90$ & $63.14 \pm 8.90$ & 0.992 \\
\hline $\mathrm{BMI}\left(\mathrm{kg} / \mathrm{m}^{2}\right)$ & $24.88 \pm 3.57$ & $24.71 \pm 2.79$ & $24.78 \pm 2.65$ & $25.54 \pm 3.18$ & 0.504 \\
\hline \multicolumn{6}{|l|}{ Past medical history } \\
\hline Malignancy & 4 & 1 & 4 & 1 & 0.022 \\
\hline Thyroid related disease & 2 & 4 & 0 & 2 & 0.488 \\
\hline Catalact & 6 & 13 & 4 & 4 & 0.112 \\
\hline Operated level & & & & & 0.300 \\
\hline L3-4 & 2 & 5 & 2 & 5 & \\
\hline$\lfloor 4-5$ & 20 & 38 & 13 & 40 & \\
\hline L5-S1 & 12 & 11 & 11 & 11 & \\
\hline Fusion type & & & & & 0.063 \\
\hline Anterior & 16 & 22 & 14 & 13 & \\
\hline Lateral & 8 & 15 & 8 & 26 & \\
\hline Posterior & 10 & 17 & 4 & 17 & \\
\hline
\end{tabular}

Values are presented as mean \pm standard deviation or number. BMI : body mass index 
come were compared among groups 1, 2, 3, and 4. For prior homogeneity tests across groups, one-way analysis of variance was used for variables with average values, and chi-square test was used for categorical values. Post hoc Tukey Honest significant difference test was used for individual comparisons of radiation exposure/screw and fluoroscopic time/screw. Statistical analysis relied on standard software (SPSS ver. 16.0 for Windows; SPSS, Inc, Chicago, IL, USA), and statistical significance was set at $p<0.05$.

\section{RESULTS}

\section{Demographics}

The results of demographic analysis are summarized in Table 1. There were no significant differences among the four groups in sexual ratio, age, BMI, operated level, and fusion type. The overall study population showed female dominance (102/170, 60\%), and average age ranged from 66.00 \pm 10.62 (group 1) to $63.46 \pm 7.49$ (group 3). Average BMI ranged from $24.71 \pm 2.79$ (group 2) to $25.54 \pm 3.18$ (group 4). A total of $5.9 \%$ (10/170) had past history of malignancy (thyroid, breast, lung, ovary, stomach, and rectus), which was significantly different across groups. A total of $4.7 \%(8 / 170)$ had thyroid disease and $15.9 \%(27 / 170)$ had cataract. The most frequently operated level was L4-5 (111/170, 65.3\%), and the most frequently used fusion approach was the anterior approach (75/170, 44.1\%).

\section{Preoperative radiologic evaluation, intraopera- tive information, and radiation exposure}

The results are summarized in Table 2. BMD ranged $-0.69 \pm$ 1.78 (group 4) to $-1.02 \pm 1.45$ (group 1) and showed osteopenia without significant differences among the four groups. A total of $14.7 \%(25 / 170)$ had sclerosis on targeted vertebral body or pedicle. Among these, 10 patients were in group 1, six patients were in group 3 , and seven patients were in group 4 . A total of $5.3 \%(9 / 170)$ had small pedicles on targeted levels, and nine of these patients were in groups 1 and 3. A total of 16.5\% (28/170) had facet hypertrophy, and 23 of these patients were in groups 1 and 3. Three variables measured in preoperative CT were significantly different among groups.

Mean operation time was significantly longer in groups 1 and 3 (50.03 and 42.08 minutes, respectively). Cement augmentation was performed in $16.5 \%$ (28/170) of the whole patient population, and the majority of these cases were in groups 1 and 2. Eleven of them were in group 1, and nine of them were in group 2, which was significantly different.

The average total radiation exposure, fluoroscopic time, radiation exposure/screw, and fluoroscopic time/screw showed significant differences across groups (Table 2). Average radiation exposure/screw was $71.45 \pm 45.75 \mu \mathrm{Sv} / \mathrm{screw}$ for group 1 , $18.77 \pm 11.51 \mu \mathrm{Sv} / \mathrm{screw}$ for group $2,19.58 \pm 7.00 \mu \mathrm{Sv} / \mathrm{screw}$ for

Table 2. Preoperative radiologic evaluation, intraoperative information, and radiation exposure

\begin{tabular}{|c|c|c|c|c|c|}
\hline & Group $1(n=34)$ & Group $2(n=54)$ & Group $3(n=26)$ & Group $4(n=56)$ & $p$-value \\
\hline \multicolumn{6}{|l|}{ Preoperative radiologic evaluation } \\
\hline BMD (T-score) & $-1.02 \pm 1.45$ & $-1.11 \pm 1.59$ & $-1.17 \pm 1.45$ & $-0.69 \pm 1.78$ & 0.464 \\
\hline Sclerosis & 10 & 2 & 6 & 7 & 0.009 \\
\hline Small pedicle & 4 & 0 & 4 & 1 & $<0.001$ \\
\hline Facet hypertrophy & 12 & 2 & 11 & 3 & $<0.001$ \\
\hline \multicolumn{6}{|l|}{ Intraoperative information } \\
\hline Operation time (minutes) & $50.03 \pm 9.00$ & $35.78 \pm 9.54$ & $42.08 \pm 9.25$ & $36.16 \pm 5.77$ & $<0.001$ \\
\hline Cement augmentation & 11 & 9 & 3 & 5 & 0.029 \\
\hline \multicolumn{6}{|l|}{ Radiation exposure } \\
\hline Radiation exposure ( $\mu S v)$ & $285.81 \pm 183.01$ & $75.09 \pm 46.05$ & $78.33 \pm 27.99$ & $17.05 \pm 11.55$ & $<0.001$ \\
\hline Fluoroscopic time (seconds) & $248.65 \pm 59.64$ & $62.67 \pm 39.36$ & $222.46 \pm 30.64$ & $86.04 \pm 50.95$ & $<0.001$ \\
\hline Radiation exposure per screw ( $\mu \mathrm{S} v / \mathrm{screw})$ & $71.45 \pm 45.75$ & $18.77 \pm 11.51$ & $19.58 \pm 7.00$ & $4.26 \pm 2.89$ & $<0.001$ \\
\hline Fluoroscopic time per screw (seconds/screw) & $62.16 \pm 14.91$ & $15.67 \pm 9.84$ & $55.62 \pm 7.66$ & $17.51 \pm 12.74$ & $<0.001$ \\
\hline
\end{tabular}

Values are presented as mean \pm standard deviation or number. BMD : bone mineral density 
group 3, and 4.26 $\pm 2.89 \mu \mathrm{Sv} / \mathrm{screw}$ for group 4. Post hoc Tukey Honest significant difference test demonstrated significant differences between each set of individual groups, except groups 2 and 3 (Fig. 4). Average fluoroscopic time/screw was $62.16 \pm 14.91$ seconds/screw for group $1,15.67 \pm 9.84$ seconds/ screw for group 2, 55.62 \pm 7.66 seconds/screw for group 3, and $17.51 \pm 12.7466$ seconds/screw for group 4. There was a significant difference in fluoroscopic time/screw between real-time imaging groups (groups 1 and 3) and intermittent imaging groups (groups 2 and 4), but there were no significant differ-

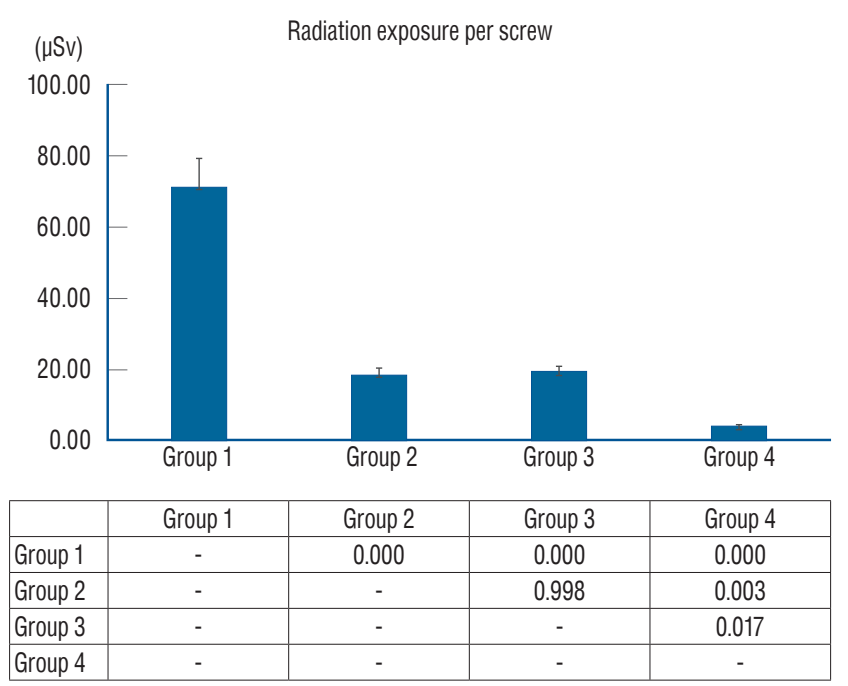

(A)

Fig. 4. Results of post-hoc comparisons using Tukey Honest significant difference test. Surgeon radiation exposure per screw is expressed in microsieverts (A), and fluoroscopic time per screw is expressed in seconds (B).

Table 3. Radiologic outcome and complication

\begin{tabular}{|c|c|c|c|c|c|c|}
\hline & $\begin{array}{l}\text { Group } 1 \text { screws } \\
(n=136)\end{array}$ & $\begin{array}{l}\text { Group } 2 \text { screws } \\
\quad(n=216)\end{array}$ & $\begin{array}{l}\text { Group } 3 \text { screws } \\
(n=104)\end{array}$ & $\begin{array}{l}\text { Group } 4 \text { screws } \\
(n=224)\end{array}$ & $p$-value & $\begin{array}{c}\text { Total } \\
(n=680)\end{array}$ \\
\hline \multicolumn{7}{|l|}{ Pedicle breach } \\
\hline Grade 0 & 135 & 214 & 103 & 223 & 0.757 & 675 \\
\hline Grade 1 & 1 & 2 & 1 & 1 & 0.901 & 5 \\
\hline Grade 2 & 0 & 0 & 0 & 0 & - & 0 \\
\hline \multicolumn{7}{|c|}{ Deviation of breach } \\
\hline \multicolumn{7}{|l|}{ Medial } \\
\hline Grade 0 & 2 & 1 & 1 & 2 & 0.834 & 5 \\
\hline Grade 1 & 0 & 0 & 0 & 0 & - & 0 \\
\hline \multicolumn{7}{|l|}{ Lateral } \\
\hline Grade 0 & 2 & 3 & 2 & 1 & 0.703 & 8 \\
\hline Grade 1 & 1 & 2 & 1 & 1 & 0.901 & 5 \\
\hline Complication & 0 & 0 & 0 & 0 & - & 0 \\
\hline
\end{tabular}

ences within groups. By changing imaging manner from realtime multiple shot to intermittent single shot, $73.7 \%$ radiation reduction was achieved in the groups without radiation modification (groups 1 and 2), and 78.2\% radiation reduction was achieved in the groups with radiation modification (groups 3 and 4). By modifying radiation source from continuous mode with standard dose to pulse mode with reduced dose, $72.6 \%$ radiation reduction was achieved in real-time imaging groups (groups 1 and 3), and 77.3\% radiation reduction was achieved in intermittent imaging groups (groups 2 and 4).

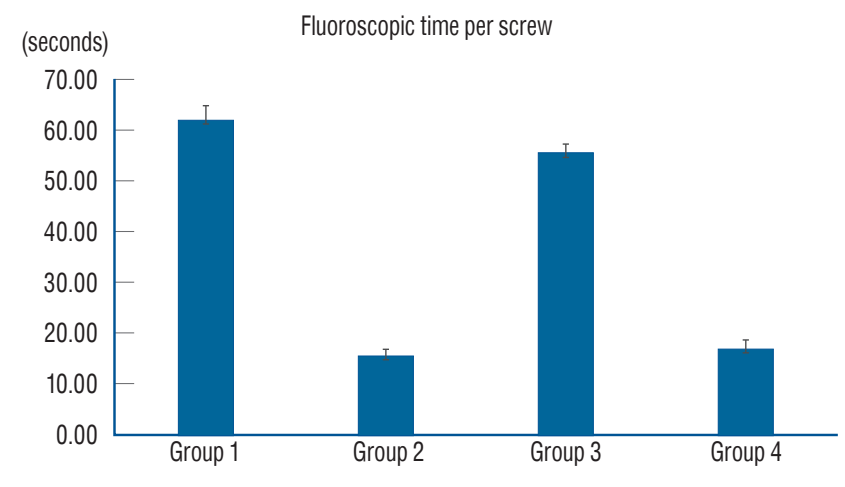

\begin{tabular}{|l|c|c|c|c|}
\hline & Group 1 & Group 2 & Group 3 & Group 4 \\
\hline Group 1 & - & 0.000 & 0.144 & 0.000 \\
\hline Group 2 & - & - & 0.000 & 0.348 \\
\hline Group 3 & - & - & - & 0.000 \\
\hline Group 4 & - & - & - & - \\
\hline
\end{tabular}

(B) microsieverts $(A)$, and fluoroscopic time per screw is expressedin seconds $(B)$. 


\section{Postoperative outcomes and complications}

The results are summarized in Table 3 . A total of 680 screws were postoperatively reviewed, and 99.3\% (675) of all inserted PPS were evaluated as grade 0 . Five screws $(0.7 \%)$ were evaluated as grade 1 : one each in groups 1,3 , and 4 , and two in group 2. There was no significant difference in pedicle breach among the groups. All five grade 1 breached screws were deviated to the lateral side. There were five grade 0 breached screws $(0.01 \%)$ deviated to the medial side and eight grade 0 breached screws $(0.02 \%)$ deviated to the lateral side from the entire study population. There was no significant difference in deviation among the groups. No patients suffered from radiculopathy due to pedicle breach or complications related to pedicle breach.

\section{DISCUSSION}

According to the results of this study, average radiation exposure/screw can be reduced $94.1 \%$ by changing the imaging manner and modifying radiation source from real-time multiple shot imaging with standard fluoroscopy setting (group 1, $71.45 \mu \mathrm{Sv} / \mathrm{screw})$ to intermittent single shot imaging with modified fluoroscopy setting (group 4, $4.26 \mu \mathrm{Sv} / \mathrm{screw}$ ). Available number of screws per year can be calculated from this result by dividing this value from annual recommended radiation exposure. This indicates that the surgeon of group 1 would insert about 280 screws (70 vertebral levels) while the surgeon of group 4 would insert about 4695 screws (1174 vertebral levels) to reach the $20 \mathrm{mSv}$ average annual limit of radiation exposure for radiation workers. The number of screws that surgeons can insert to maintain the average annual radiation exposure limit is about 1062 (265 vertebral levels) for group 2 and 1021 (255 vertebral levels) for group 3. This study demonstrated that reduction of intraoperative radiation can be simply achieved by changing the imaging manner and fluoroscopy settings from continuous mode to pulse mode with minor $\mathrm{kV}$ reduction. In addition, only five pedicle screws were breached greater than $2 \mathrm{~mm}$, and they did not cause any radiculopathy symptoms. There was no significant difference in pedicle breach rate among the groups. Therefore, the overall effective breach rate was $0 \%$. The results also indicate that the procedure can be performed safely even in groups with modified radiation.
Image quality should be appropriately maintained for surgeons to perform PPS procedures and avoid any complications caused by mal-positioning of the bone needle or wire during the procedure or pedicle breach. The $\mathrm{kV}$ is the energy of photons, and $\mathrm{mA}$ is the quantity of photons ${ }^{11)}$. The $\mathrm{kV}$ is related to image brightness while $\mathrm{mA}$ is related to image resolution. The optimum current setting of conventional fluoroscopy is suggested as high $\mathrm{kV}$ and low $\mathrm{mA}$, and most standard settings of fluoroscopy machines follow this principle. Lai et al. ${ }^{10)}$ demonstrated that all relevant anatomical structures were identified on lateral lumbar spine radiographs despite the use of low-dose protocols and suggested that the optimal settings were $95 \mathrm{kV}$ and $4.5 \mathrm{~mA}$; radiation exposure increased by decreasing $\mathrm{kV}$ and increasing $\mathrm{mA}$. However, baseline current depends on the weight and BMI of every patient. Therefore, we suggest that minor reduction of $\mathrm{kV}$ without increased $\mathrm{mA}$ is the most appropriate current setting to reduce radiation while minimizing changes in image quality. To overcome image quality issues, software that enhances imaging quality after dose reduction has been introduced, such as LessRay (NuVasive, Inc., San Diego, CA, USA) ${ }^{21)}$. Godzik et al. ${ }^{6)}$ performed interbody fusion under ultra-low radiation imaging and enhanced image quality using LessRay image enhancement technology. They achieved $65.8 \%$ to $81.0 \%$ reduction in radiation exposure by this technique. Nayar et al. ${ }^{15)}$ evaluated pedicle screw placement accuracy with this technique and reported that it did not compromise accuracy compared to conventional fluoroscopy; meanwhile, it significantly decreased radiation exposure for patients and operating room personnel.

Since PPS insertion mostly relies on fluoroscopic images, it is more difficult to perform the procedure for patients with factors that lower image quality or who have abnormal anatomic features around the screw insertion point. This leads to more frequent fluoroscopic imaging during the procedure, which is also related to more radiation exposure for surgeons and patients. In this study, preoperative anatomic features such as sclerosis, pedicle size, and facet joint hypertrophy were measured on preoperative CT. These features were more frequently observed in groups 1 and 3 with statistical significance $(p=0.009)$. Patients with sclerosis require additional work with a rigid tool to prepare the pathway for the screw. Patients with small pedicles require more frequent AP and lateral confirmation during pedicle penetration with the bone needle, tapper, and real screw. Facet joint hypertrophy that 
covers the insertion points interferes with the natural slope between the transverse process and superior articular process, which requires more frequent fluoroscopic confirmation. Consequently, operation time and fluoroscopic time per screw were significantly longer in patients with these anatomic features (groups 1 and 3). High BMI was also reported as a factor that prolongs fluoroscopic time $e^{17)}$. Higher required current setting is also expected to obtain the same image quality with increased BMI. However, BMI of the four groups was not significantly different, and the gap between the highest (group 4, 25.54 ) and lowest value (group 2, 24.71) was only 0.83. Therefore, BMI did not influence differences in radiation exposure among the groups in this study. Although reduced radiation exposure is beneficial to all people in the operation room, realtime multiple shot imaging with standard dose should be considered in patients with factors that possibly jeopardize safe PPS insertion. This will help to avoid screw misplacement and prevent revisional surgery.

There are also other recommendations for reducing radiation exposure. Yoshihara and Paulino ${ }^{22)}$ performed pediatric scoliosis surgery with an average fusion level of 11 mostly under AP views and were positioned a few feet away from the Xray tube during lateral view inspection. By increasing distance and maintaining AP views, they achieved lower radiation exposure than Bindal et al. ${ }^{2}$, who performed 1 or 2 level transforaminal interbody fusion ${ }^{12)}$. Standing opposite to the X-ray source during lateral imaging and positioning the X-ray source below the patient were also recommended to reduce radiation scatter. Lee et al. ${ }^{13)}$ reported that standard and translateral C-arm configurations caused lower scatter doses to sensitive organs than inverted and trans-lateral C-arm configurations in their study using a whole body anthropomorphic phantom. For hand protection, a hands-off technique or the use of long instruments to hold the pedicle screw engaging devise is recommended ${ }^{18}$. Because eye lenses are the most sensitive organs to radiation exposure, turning heads 90 degrees away from patients during imaging also reduces radiation scatter to the eyes ${ }^{13}$. Tight wearing of thyroid shields or bismuth masking reagent significantly reduced radiation exposure compared to wearing shields loosely ${ }^{1)}$. Unlike other protective strategies, such as shielding, distancing, positioning, and $\mathrm{C}$-arm configuration, modification of imaging manner, mode, and dose of fluoroscopy reduces radiation for both surgeons and patients. Yoshihara and Paulino ${ }^{22)}$ reported that ex- posure of patient organs without direct radiation, such as thyroids and gonads, was not significant. However, patients with underlying radiosensitive diseases, such as cataract (15.9\%), malignancy (5.9\%), and thyroid disease (4.7\%), require more delicate control and protection. Surgeons must apply intermittent single shot imaging when possible and understand methods to modify radiation source to reduce radiation exposure in patients with these diseases.

Many other spinal procedures require fluoroscopic guidance. Ahn et al. ${ }^{1)}$ reported that unprotected radiation exposure during percutaneous endoscopic lumbar discectomy (PELD) was $785 \mu \mathrm{Sv} / \mathrm{case}$ on the neck, $171 \mu \mathrm{Sv} / \mathrm{case}$ on the chest, 732 $\mu \mathrm{Sv} / \mathrm{case}$ on the left ring finger, and $669 \mu \mathrm{Sv} / \mathrm{case}$ on the right ring finger. They strongly suggested wearing protective lead collars and aprons to reduce radiation dose by $96.9 \%$ and $94.2 \%$, respectively. Average operation time and fluoroscopic time were $49.8 \pm 15.0$ minutes and $150 \pm 66$ seconds/case, respectively. $\mathrm{Choi}^{4)}$ reported that the lowest radiation exposure during kyphoplasty (KP) was measured when the X-ray tube was away from the surgeon and beneath the bed. The average radiation exposure in 24 cases was $298 \mu \mathrm{Sv} / \mathrm{case}$ on the head, $282 \mu \mathrm{Sv} / \mathrm{case}$ on the neck, $971 \mu \mathrm{Sv} / \mathrm{case}$ on the chest, $897 \mu \mathrm{Sv} /$ case on the abdomen, and $816 \mu \mathrm{Sv} / \mathrm{case}$ on the knee. Average operation time and fluoroscopic time were 16.3 minutes and 457.8 seconds/case, respectively. The reported radiation exposure of the chest and fluoroscopic time of PELD were lower than that of group 1 in our study $285 \mu \mathrm{Sv} / \mathrm{case}$ and $248.6 \mathrm{sec}-$ onds/case, respectively. However, radiation exposure of the chest and fluoroscopic time of KP exceed those of group 1 in our study. According to the reported fluoroscopic time, it can be assumed that procedures in these studies were performed with real-time multiple shot imaging. This indicates that radiation exposure could be reduced by modifying imaging manner and mode radiation source. Although operation time was shorter with KP than with other procedure types, KP showed longer fluoroscopic time, which leads to larger radiation exposure. Although annual average or maximal limits were set to avoid deterministic effects of radiation exposure, any dose above zero can increase the radiation-induced cancer risk by the cumulative effect, also known as the stochastic effect. Evidence has demonstrated that the average risk for radiation-induced cancer in the general population is $5 \%$ per $\mathrm{Sv}^{8)}$. Therefore, spine surgeons should maintain radiation exposure "as low as reasonably achievable", as ICRP recommends, not only 
for themselves but also for patients. Modification of imaging manner and mode of radiation source can be applied to any procedure using fluoroscopic guidance and will contribute to reduce the overall radiation exposure of spine surgeons.

To the author's knowledge this is the first study demonstrated the radiation exposure reduction effect of radiation source modification with pulse mode during the PPS procedure. However this study involves some limitations. First, selection and recall bias were possible due to the retrospective nature of this study. Analyzing risk factors for changing imaging manner and the mode of radiation source are also significant aspects. However, it couldn't be achieved because patients were designated to each group retrospectively without documenting the prior imaging manner and fluoroscopy setting. Although some preoperative radiologic features were indicated as candidate risk factors, prospective study design is needed for the identification of the risk factors in near future. Despite the exclusion of the first 40 cases, data interpretation is still susceptible to the surgeon's learning curve bias. Third, measurements of radiation dose were limited to the anterior chest area of the surgeon. Radiation exposure for other body parts was not measured. Radiation exposure was also not measured on other medical personnel or patients. Fourth, pedicle breach was evaluated on postoperative MRI, which contains artifacts that may compromise accurate evaluation. Finally, clinical outcomes such as postoperative pain, disability, and satisfaction were not assessed. Despite these limitations, this study proves decreased radiation exposure with modification of imaging manner, mode, and dose of fluoroscopy.

\section{CONCLUSION}

The average radiation exposure/screw of a spine surgeon can be reduced $94.1 \%$ by modifying the imaging manner and radiation source from real-time multiple shot imaging with continuous mode to intermittent single shot imaging with pulse mode and reducing the $\mathrm{kV}$ setting. The radiation exposure of spine surgeons, operative staff, and patients should be as low as reasonably achievable to avoid the stochastic effect of radiation exposure. These modifications can be instantly applied to any procedure using fluoroscopic guidance and will contribute to reduced overall radiation exposure of spine surgeons.

\section{CONFLICTS OF INTEREST}

No potential conflict of interest relevant to this article was reported.

\section{INFORMED CONSENT}

Informed consent was obtained from all individual participants included in this study.

\section{AUTHOR CONTRIBUTIONS}

\author{
Conceptualization : HJK, ESP \\ Data curation : HJK \\ Formal analysis : $\mathrm{HJK}$ \\ Funding acquisition : CHP, SHL \\ Methodology: HJK, SWC \\ Project administration : CHP, SHL \\ Visualization : HJK \\ Writing - original draft : HJK \\ Writing - review \& editing : HJK, ESP, SWC
}

\section{ORCID}

$\begin{array}{ll}\text { Hyun Jun Kim } & \text { https://orcid.org/0000-0002-2489-7524 } \\ \text { Eun Soo Park } & \text { https://orcid.org/0000-0002-1628-5296 } \\ \text { Sang Ho Lee } & \text { https://orcid.org/0000-0002-8526-0260 } \\ \text { Chan Hong Park } & \text { https://orcid.org/0000-0002-1305-0395 } \\ \text { Seok Won Chung } & \text { https://orcid.org/0000-0002-7577-0603 }\end{array}$

\section{References}

1. Ahn Y, Kim CH, Lee JH, Lee SH, Kim JS : Radiation exposure to the surgeon during percutaneous endoscopic lumbar discectomy: a prospective study. Spine (Phila Pa 1976) 38 : 617-625, 2013

2. Bindal RK, Glaze S, Ognoskie M, Tunner V, Malone R, Ghosh S : Surgeon and patient radiation exposure in minimally invasive transforaminal lumbar interbody fusion. J Neurosurg Spine 9 : 570-573, 2008

3. Braun E, Sack AM, Sayed D, Manion S, Hamm B, Brimacombe M, et al. : Reducing radiation exposure in lumbar transforaminal epidural steroid injections with pulsed fluoroscopy: a randomized, double-blind, con- 
trolled clinical trial. Pain Physician 21 : 53-60, 2018

4. Choi HC : Fluoroscopic radiation exposure during percutaneous kyphoplasty. J Korean Neurosurg Soc 49 : 37-42, 2011

5. Gautschi OP, Schatlo B, Schaller K, Tessitore E : Clinically relevant complications related to pedicle screw placement in thoracolumbar surgery and their management: a literature review of 35,630 pedicle screws. Neurosurg Focus 31 : E8, 2011

6. Godzik J, Nayar G, Hunter WD, Tumialán LM : Decreasing radiation emission in minimally invasive spine surgery using ultra-low-radiation imaging with image enhancement: a prospective cohort study. World Neurosurg 122 : e805-e811, 2019

7. Gupta G, Singh R, Kotasthane DS, Kotasthane VD : Myelodysplastic syndromes/neoplasms: recent classification system based on World Health Organization classification of tumors - International Agency for Research on Cancer for Hematopoietic and Lymphoid Tissues. J Blood Med 1 : 171-182, 2010

8. Hall EJ : Lessons we have learned from our children: cancer risks from diagnostic radiology. Pediatr Radiol 32 : 700-706, 2002

9. Hou BB, Yao Y, Wu LM, Qiao Y, Zheng LH, Ding LG, et al. : Optimized fluoroscopy setting and appropriate project position can reduce $X$-ray radiation doses rates during electrophysiology procedures. Chin Med J (Engl) $128:$ 1151-1153, 2015

10. Lai ZH, Sá Dos Reis C, Sun Z : Effective dose and image optimisation of lateral lumbar spine radiography: a phantom study. Eur Radiol Exp 4 : 13, 2020

11. Lakhwani OP, Dalal V, Jindal $M$, Nagala A : Radiation protection and standardization. J Clin Orthop Trauma 10 : 738-743, 2019

12. Lee K, Lee KM, Park MS, Lee B, Kwon DG, Chung CY : Measurements of surgeons' exposure to ionizing radiation dose during intraoperative use of C-arm fluoroscopy. Spine (Phila Pa 1976) 37 : 1240-1244, 2012

13. Lee SY, Min E, Bae J, Chung CY, Lee KM, Kwon SS, et al. : Types and arrangement of thyroid shields to reduce exposure of surgeons to ionizing radiation during intraoperative use of C-arm fluoroscopy. Spine (Phila
Pa 1976) $38:$ 2108-2112, 2013

14. Lin EC : Radiation risk from medical imaging. Mayo Clin Proc 85 : 1142-1146; quiz 1146, 2010

15. Nayar G, Blizzard DJ, Wang TY, Cook S, Back AG, Vincent D, et al. : Pedicle screw placement accuracy using ultra-low radiation imaging with image enhancement versus conventional fluoroscopy in minimally invasive transforaminal lumbar interbody fusion: an internally randomized controlled trial. J Neurosurg Spine 28 : 186-193, 2018

16. Richards PJ, George J, Metelko M, Brown M : Spine computed tomography doses and cancer induction. Spine (Phila Pa 1976) 35 : 430-433, 2010

17. Smuck M, Zheng P, Chong T, Kao MC, Geisser ME : Duration of fluoroscopic-guided spine interventions and radiation exposure is increased in overweight patients. PM R 5 : 291-6; quiz 296, 2013

18. Srinivasan D, Than KD, Wang AC, La Marca F, Wang PI, Schermerhorn TC, et al. : Radiation safety and spine surgery: systematic review of exposure limits and methods to minimize radiation exposure. World Neurosurg 82 : 1337-1343, 2014

19. Valentin J, Alexakhin RM, Cousins C, et al. : The 2007 recommendations of the international commission on radiological protection. Orlando : Elsevier, 2007

20. Wang H, Zhou Y, Li C, Liu J, Xiang L : Comparison of open versus percutaneous pedicle screw fixation using the sextant system in the treatment of traumatic thoracolumbar fractures. Clin Spine Surg 30 : E239E246, 2017

21. Wang TY, Farber SH, Perkins SS, Back AG, Byrd SA, Chi D, et al. : Internally randomized control trial of radiation exposure using ultra-low radiation imaging versus traditional C-arm fluoroscopy for patients undergoing single-level minimally invasive transforaminal lumbar interbody fusion. Spine (Phila Pa 1976) 42 : 217-223, 2017

22. Yoshihara $H$, Paulino $C B$ : Radiation exposure to the surgeons and patients in fluoroscopic-guided segmental pedicle screw placement for pediatric scoliosis. Spine (Phila Pa 1976) 43 : E1398-E1402, 2018 